EESTI NSV TEADUSTE AKADEEMIA TOIMETISED 1955. IV kd., nr. 2 ИЗВЕСТИЯ АКАДЕМИИ НАУК ЭСТОНСКОИ ССР 1955. ТОМ IV, № 2

\title{
ИССЛЕДОВАНИЕ ЦЕНТРОВ ЗАХВАТА В КРИСТАЛЛАХ МЕТОДОМ ТЕРМИЧЕСКОГО ОБЕСЦВЕЧИВАНИЯ *
}

\author{
ч. Б. ЛУЩик, \\ кандидат физико-математических наук
}

\section{Введение}

В настоящее время основным методом исследования центров захвата в кристаллофосфорах является метод термического высвечивания. Огромная чувствительность метода и большая наглядность получаемых результатов обеспечили этому методу самое широкое применение. В послевоенные годы методом термовысвечивания получен ряд ценных данных о центрах захвата в различных фосфорах. Однако одновременно были вскрыты некоторые недостатки этого метода, часть из которых носит принципиальный характер.

Действительно, поскольку метод термовысвечивания существенно связан с регистрацией яркости свечения, то он, естественно, не может быть применен для исследования центров захвата в несветящихся объектах. Это большой недостаток метода. Отметим, например, что по этой причине мы не можем исследовать методом термовысвечивания центры захвата чистого основного вещества и строго решить вопрос о роли активатора в создании центров захвата для электронов. Более того, даже в кристаллофосфорах наличие температурного тушения не позволяет в ряде случаев исследовать глубокие уровни захвата.

Очевидно, что для преодоления этих трудностей, возникающих при применении метода термовысвечивания, следует отказаться от регистрации яркости свечения и перейти к измерению каких-то других величин, связанных с освобождением электронов из центров захвата.

Можно измерять, например, зависимость проводимости от температуры при равномерном нагреве возбужденного кристалла. Такой метод исследования центров захвата («метод термического освобождения заряда») получил в последние годы некоторое распространение $\left({ }^{1-4}\right)$. Однако из-за ряда экспериментальных трудностей, возникающих при измерении проводимости кристалла, а также из-за отсутствия строгой теории этот метод широкого применения не получил.

Представляется целесообразным использовать еще один метод, во многом подобный методу термического высвечивания и методу термического оєвобождения заряда. Известно, что при возбуждении кристалл приобре-

* Доложено на семинаре Физического института им. П. Н. Лебедева Академии наук СССР 9 марта 1955 г. 
тает «добавочное поглощение», поглощение на электронных и дырочных центрах захвата. Хорошо известно также, что при достаточно сильном нагреве возбужденного кристалла это добавочное поглощение пропадает. Если регистрировать зависимость коэффициента поглощения в максимуме одной из полос добавочного поглощения $\Delta x_{m i}$ от температуры $T$ при равномерном нагреве возбужденного кристалла, то в некоторой довольно узкой области температур $\Delta \varkappa_{m i}(T)$ будет иметь резкий спад, обусловленный тепловой ионизацией соответствующих центров захвата.-Очевидно, что положение этого спада должно характеризовать глубину уровней захвата. Так как в ряде случаев уничтожение добавочного поглощения связано с исчезновением окраски кристалла, то метод исследования центров захвата'по зависимости $\Delta \varkappa_{m i}(T)$ можно назвать методом термического обесцвечивания.

\section{§ 1. Теоретические основы метода}

Коэффициент поглощения в определенной полосе добавочного поглощения пропорционален числу электронов (дырок), запасенных на данных уровнях захвата $n_{i}$ :

$$
\Delta \varkappa_{m i}=B_{i} \cdot n_{i}
$$

Коэффициент пропорпиональности $B_{i}$, вообще говоря, зависит от температуры. Однако эта зависимость, связанная с изменением формы и положения полосы поглощения, весьма слаба по сравнению с чрезвычайно резкой зависимостью $n_{i}(T)$. Из экспериментальных данных и теоретических расчетов следует $\left({ }^{5}\right)$, что

$$
B_{i} \sim T^{-0,5}
$$

Следовательно, можно зависимостью $B_{i}(T)$ пренебречь и считать, что зависимость $\Delta \varkappa_{m i}(T)$ сводится к зависимости $n_{i}(T)$. Последняя же может быть легко получена.

Если в кристалле имеются уровни захвата нескольких сортов, значительно различающиеся по глубине, и если кинетика послесвечения носит бимолекулярный характер, то изменение числа электронов на $i$-уровнях захвата будет определяться следующим уравнением

$$
-\frac{d n_{i}}{d t}=p_{i} \cdot n_{i} \frac{\sigma_{p} \cdot n_{i}+\sum_{\sigma_{p} \cdot n_{i}}+\sigma_{3 i}\left(N_{i}-n_{i}\right)+\sum_{j>i} \sigma_{3 j}\left(N_{j}-n_{j}\right)}{.}
$$

Здесь $p_{i}=p_{0 i} \exp \left(-E_{T i} / k T\right)^{-}-$вероятность тепловой ионизации центров захвата, $\sigma_{p}$ и $\sigma_{3}$ - эффективные сечения рекомбинации и захвата, $N_{i}$ - число $i$-уровней захвата.

Член $2 \sigma_{3 j^{\prime}} N_{j}-n_{j}$ ) характеризует вероятность повторных захватов элек$j>i$

тронов, освобождающихся с $i$-уровней захвата, на более глубоких уровнях.

Если исследуется зависимость $n_{i}(T)$ для наиболее глубоких уровней захвата, то $\Sigma \sigma_{3 j}\left(N_{j}-n_{j}\right)=0$, и из соотношения (3) имеем

$$
j>i
$$

$$
-\frac{d n_{i}}{d t}=p_{i} \cdot n_{i} \frac{\sigma_{p} \cdot n_{i}}{\sigma_{p} \cdot n_{i}+\sigma_{3 i}\left(N_{i}-n_{i}\right)} .
$$


Легко видеть, что при этом в случае большой вероятности повторных захватов

$$
A_{3}=\sigma_{s i}\left(N_{i}-n_{i}\right) \gg \sigma_{p} \cdot n_{i}=A_{p}
$$

в условиях равномерного нагрева со скоростью $\beta_{0}=d T / d t$ соотношение (4) дает

$$
n_{i}=\frac{n_{0 i}}{\left(1+\frac{\sigma_{p} \cdot n_{0 i} \cdot p_{0 i}}{\sigma_{3} \cdot N_{i} \cdot \beta_{0}} \int_{T_{i}}^{T_{z}}\left[\exp \left(-E_{T i}{ }^{\prime} k T\right)\right] d T\right)} .
$$

В случае же малой вероятности повторных захватов

из соотношения (4) имеем

$$
\sigma_{3 i}\left(N_{i}-n_{i}\right) \ll \sigma_{p} \cdot n_{i}
$$

$$
n_{i}=n_{i 0} \exp \left\{-\frac{p_{0 i}}{\beta_{0}} \int_{T_{t}}^{T_{2}}\left[\exp \left(-E_{T i} / k T\right)\right] d T\right\}
$$

Теоретические кривые термического обесцвечивания для крайних случаев $A_{3} \gg A_{p}$ и $A_{3} \ll A_{p}$, рассчитанные по соотношениям (6) и (8) для разных величин тепловых энергий ионизации центров захвата $E_{T i}$, приведены на рис. 1 и 2 . Қак и следовало ожидать, в некотором узком интервале температур наблюдается резкий спад $n_{i}(T)$. Положение этого спада естественно характеризовать температурой $T_{k}$, при которой $n_{i}=0,5 \cdot n_{0 i}$. В качестве второго параметра кривой термообесцвечивания удобно выбрать «полуширину спада» $\delta_{k}=-n_{i k} /\left(\frac{d n_{i k}}{d T}\right)_{T k}$. Графический смысл этой величины ясен из рис. 1.

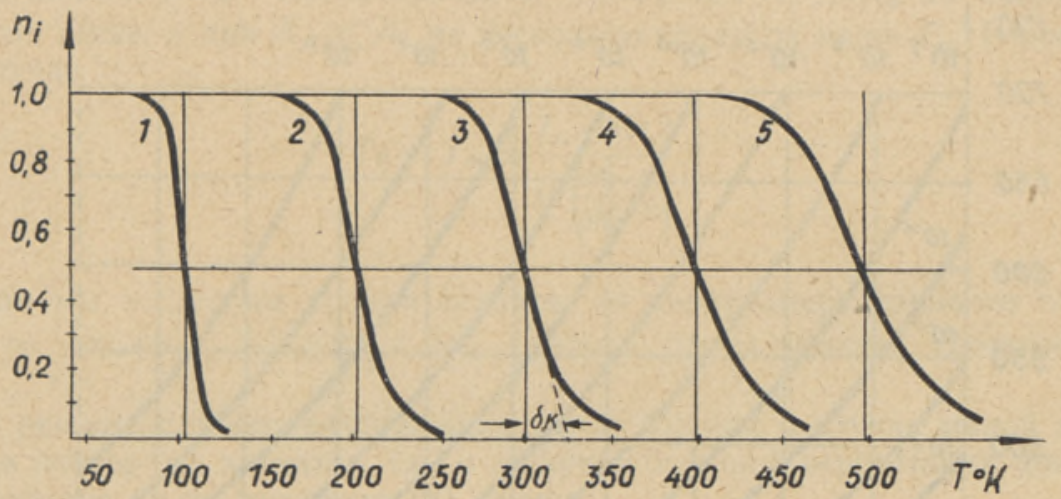

Рис. 1. Теоретические кривые термического обесцвечивания для случая большой вероятности повторных захватов. $p_{0}=10^{10} \mathrm{ceK}^{-1}, \beta_{0}=0,15$ град/сек, $\sigma_{p} n_{i o} / \sigma_{3} N_{i}=0,01 . \quad$ 1. $E_{T}=0,2$ эв, $\quad$ 2. $E_{T}=0,4$ эв, 3. $E_{T}=0,6$ эв, 4. $E_{T}=0,8$ эв, 5. $E_{T}=1,0$ эв.

Опыт показал, что величины $T_{k}$ и $\delta_{k}$ могут быть легко определены из экспериментальных данных. Как следует из рис. 1 и 2 , с большой степенью точности соблюдаются соотношения

$$
\begin{aligned}
& T_{k}=\alpha \cdot E_{T}, \\
& \delta_{k}=\alpha^{\prime} \cdot E_{T} .
\end{aligned}
$$


Коэффициенты пропорциональности $\alpha$ и $\alpha^{\prime}$ существенно зависят от $\beta_{0}$, $p_{0}, \sigma_{p} / \sigma_{3}, n_{0} / N$. Эта зависимость может быть легко получена в аналитическом виде. Если имеет место соотношение (4), то в общем случае

$$
T_{k}=\frac{E_{T}}{k} \frac{1}{\ln \left[\frac{p_{0}}{\beta_{0}} \delta_{k}\left(1+\frac{2 \sigma_{3} N}{\sigma_{p} \cdot n_{0}}-\frac{\sigma_{3}}{\sigma_{p}}\right)^{-1}\right]} .
$$

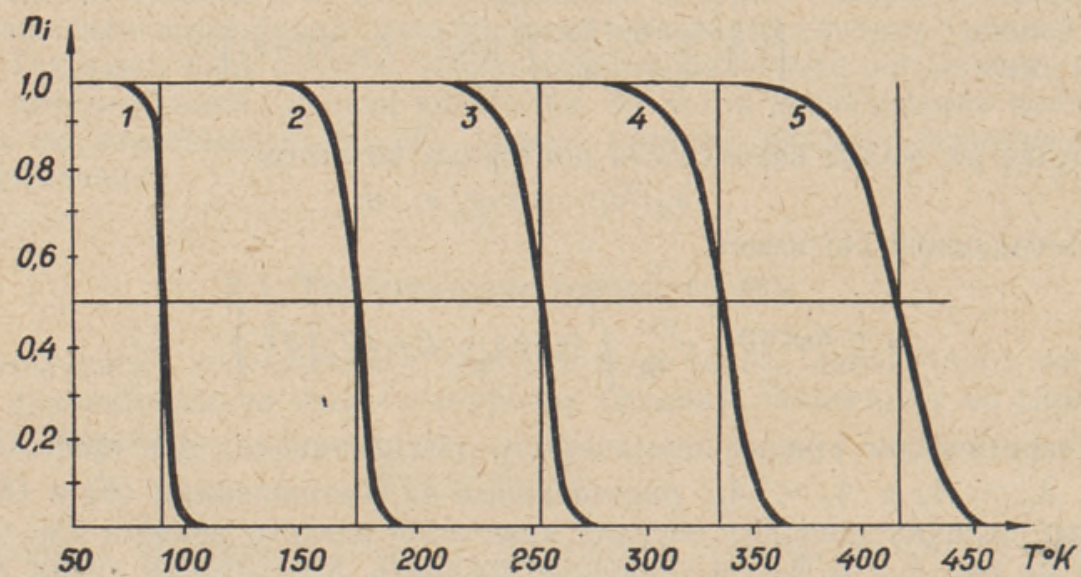

Рис. 2. Теоретические кривые термического обесцвечивания для случая малой вероятности повторных захватов. $p_{0}=10^{10}$ сек $^{-1}, \beta_{0}=0,15$ град/сек.
1. $E_{T}=0,2$ эв,
2. $E_{T}=0,4$ эв,
3. $E_{T}=0,6$ эв.
4. $E_{T}=0,8$ эв,

$$
\text { 5. } E_{T}=1,0 \text { эв. }
$$

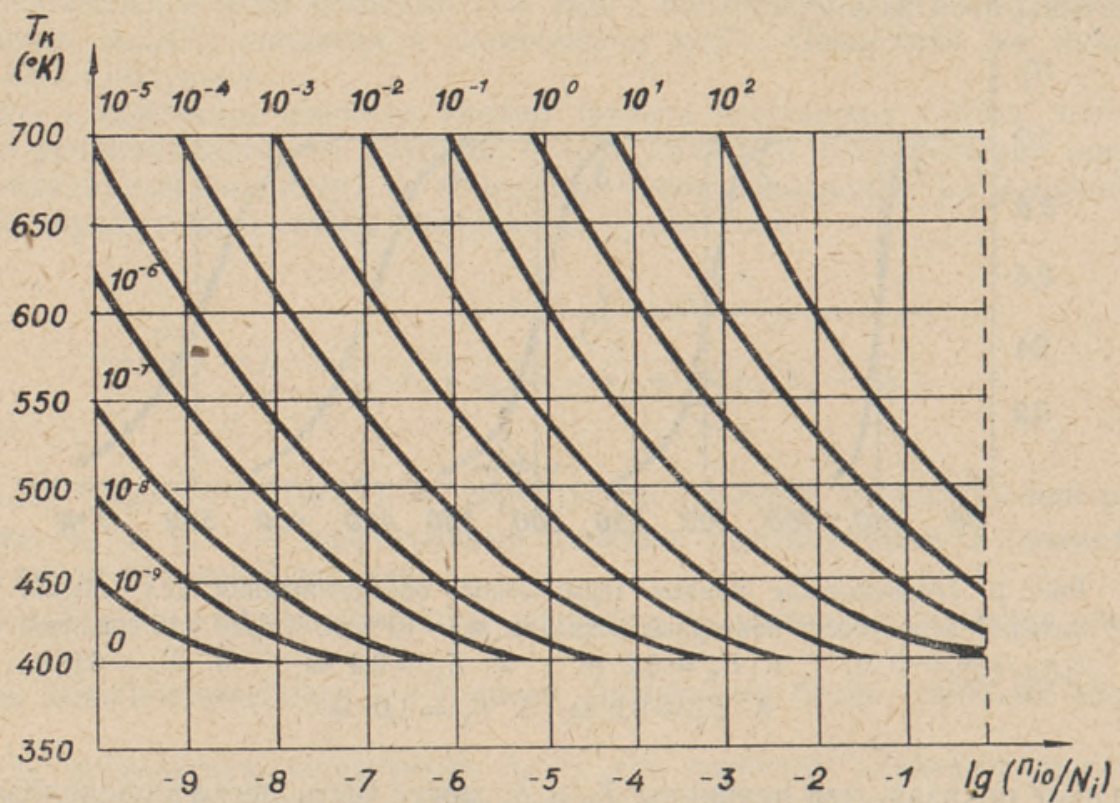

Рис. 3. Теоретнческая зависимость $T_{k}$ от числа запасенных электронов при различных $\gamma=\sigma_{3} / \sigma_{p}\left(E_{T}=1,0\right.$ эв, $p_{0}=10^{10} \mathrm{ce \kappa}^{-1}, \beta_{0}=0,15 \mathrm{rрaд} /$ сек,

$$
\left.\delta_{k}=20^{\circ} \mathrm{K}\right) \text {. }
$$


Из соотношения (11) следует, что $T_{k}$ изменяется симбатно с изменением $\beta_{0}$ и антисимбатно с изменением $p_{0}$. Особый интерес представляет зависимость $T_{k}$ от числа запасенных электронов. Эта зависимость, рассчитанная по соотношению (11), приведена на рис. 3. Қаждая кривая соответствует определенной величине параметра $\gamma=\sigma_{3} / \sigma_{p}$. Из рис. 3 следует, что в случае полного отсутствйя повторных захватов $T_{k}$ не зависит от числа запасенных электронов. В случае же $\sigma_{3} \neq 0, T_{k}=T_{k}\left(n_{0}\right)$; если $\sigma_{3}>\sigma_{p}, T^{0}{ }_{k}$ зависит от $n_{0}$ при любых значениях $n_{0}$; если же $\sigma_{3}<\sigma_{p}$, то $T_{k}$ начинает зависеть от $n_{0}$ только при достаточно малых значениях $n_{0}$, когда соблюдается соотношение $A_{3} \gg A_{p}$.

Таким образом, исследуя зависимость $T_{k}\left(n_{0}\right)$ по величине $n_{0}$, при которой начинается ощутимое изменение $T_{k}$, можно оценить величину $\sigma_{s} / \sigma_{p}$.

Если имеет место соотношение (4), то в общем случае

$$
\delta_{k}=\frac{E_{T}}{k} \cdot \frac{1}{\left\{\ln \left[\frac{p_{0}}{\beta_{0}} \delta_{k}\left(1+\frac{2 \sigma_{3} N}{\sigma_{p} \cdot n_{0}}-\frac{\sigma_{3}}{\sigma_{p}}\right)^{-1}\right]\right\}^{2}} \frac{1+\frac{4 \sigma_{3} N}{\sigma_{p} \cdot n_{0}}-\frac{\sigma_{3}}{\sigma_{p}}}{1+\frac{2 \sigma_{3} N}{\sigma_{p} \cdot n_{0}}-\frac{\sigma_{3}}{\sigma_{p}}} .
$$

Таким образом, $\delta_{k}$ существенно зависит от $p_{0}, \beta_{0}, \sigma_{p} / \sigma_{3}$. Особенно интересна зависимость $\delta_{k}$ от $n_{0}$. В случае отсутствия повторных захватов $\delta_{k} \neq \delta_{k}\left(n_{0}\right)$. Если же вероятность повторных захватов велика, то при уменьшении $n_{0}$ полуширина спада должна увеличиваться.

Следует отметить, что дифференцированием из $n_{i}(T)$ получается кривая $n_{i}^{\prime}(T)$, которая для наиболее глубоких уровней захвата должна совпадать с кривой термовысвечивания $I(T)$. Положение максимума последней $T_{m}$ близко к $T_{k}$. Легко показать, что $T_{k}$ при $A_{3} \gg A_{p}$ на несколько градусов выше, а при $A_{3} \ll A_{p}$ на несколько градусов ниже $T_{m}$. В последнем случае

$$
T_{k}-T_{m} \cong \frac{T_{m} \cdot \ln \ln 2}{\ln \left(\frac{p_{0}}{\beta_{0}} \delta_{k}\right)}
$$

Если мы исследуем уровни захвата, не являющиеся наиболее глубокими, то $\underset{j>i}{\sum} \sigma_{3 j}\left(N_{j}-n_{j}\right) \neq 0$, и мы должны рассматривать соотношение

(3). В режиме слабого возбуждения $\left(n_{j} \ll N_{j}\right)$ как в случае малой вероятности повторных захватов, так и в случае большой вероятности повторных захватов из соотношения (3) имеем

$$
n_{i}=n_{i 0} \exp \left\{-\frac{C p_{0 i}}{\beta_{0}} \int_{T_{1}}^{T}\left[\exp \left(-E_{T_{i}} / k T\right)\right] d T\right\}
$$

$$
\begin{array}{lc}
\text { Для } A_{3} \ll A_{p} & C=1 . \\
\text { Для } A_{3} \gg A_{p} & C=\frac{\sum_{j>i} \sigma_{3 j} N_{j}}{\sigma_{3 i} N_{i}+\sum_{j>i} \sigma_{3 j} N_{j}} .
\end{array}
$$




$$
T_{k}=\frac{E_{T}}{k} \frac{1}{\ln \left(C \frac{p_{0}}{\beta_{0}} \delta_{k}\right)}
$$

и

$$
\delta_{k}=\frac{E_{T}}{k} \frac{1}{\left[\ln \left(C \frac{p_{0}}{\beta_{0}} \delta_{k}\right)\right]^{2}}
$$

Таким образом, для всех уровней захвата, кроме наиболее глубоких, $T_{k}$ и $\delta_{k}$ не зависят от числа запасенных электронов.

До сих пор мы полагали, что уменьшение числа электронов на уровнях захвата происходит за счет их теплового освобождения в зону проводимости и последующей рекомбинации с дырками, находящимися на дырочных центрах захвата. Очевидно, однако, что уменьшение числа запасенных электронов может происходить и вследствие тепловой ионизации дырочных центров захвата с последующей рекомбинацией дырок с электрснами, находящимися на уровнях захвата. При этом зависимость $n_{i}(T)$ для электронов будет определяться тепловой энергией ионизации дырочных центров захвата. Законы изменения числа электронных и числа дырочных центров захвата с температурой должны быть при этом совершенно одинаковы. Если тепловая энергия ионизации дырочных центров захвата $Q_{T}$ меньше $E_{T}$, то по $n_{i}(T)$ определить $E_{T}$ невозможно. Если же $E_{T}<Q_{T}$, то зависимость $n_{i}(T)$ дает нам величину $E_{T}$, но не позволяет определить $Q_{T}$. Следовательно, по зависимости $n_{i}(T)$ может быть найдена только меньшая из величин $E_{T}$ и $Q_{T}$.

\section{§ 2. Экспериментальное получение кривых термообесцвечивания}

Для экспериментального получения кривых термического обесцвечивания мы использовали специально приспособленный для этой цели фотоэлектрический спектрофотометр СФ-4. Нагрев осуществлялся со строго постоянной скоростью $\beta_{0}=0,15$ град/сек.

Для того чтобы в процессе измерения величины $\Delta \varkappa_{m i}$ уменьшение числа запасенных электронов за счет их оптического освобождения было пренебрежимым, следует работать при достаточно слабых световых потоках, направляемых на исследуемый кристалл. Очевидно, что это требование связано только с чувствительностью используемых приемников радиации и всегда может быть удовлетворено, Для исследованных нами кристаллов при измерениях на спектрофотометре СФ-4 число оптически освобождаемых электронов не превышало $1 \%$ от числа электронов, освобождаемых тепловым движением.

При измерении кривых термообесцвечивания светящихся кристаллов перед окном фотоэлемента помещались соответствующие светофильтры, пропускающие только «рабочую длину волны», соответствующую максимуму полосы добавочного поглощения *.

Наиболее благоприятными объектами для применения метода термообесцвечивания являются монокристаллы, допускающие прецизионные

* В первоначальных опытах свечение кристаллов нами не учитывалось, что приводило к появлению на кривой $A x_{m i}(T)$ перегибов и даже максимумов, соответствующих пикам термовысвечивания исследуемых кристаллов. 
измерения коэффициента поглощения. Однако, так как измерения термообесцвечивания производятся на одной длине волны, то этим методом могут быть исследованы и тонкие поликристаллические слои. Очевидно, что при этом наибольшие удобства для исследования представляют так называемые сублимат-фосфоры $\left({ }^{6}\right)$.

Следует отметить, что чувствительность метода термообесцвечивания значительно ниже чувствительности метода термовысвечивания. Последний метод позволяет исследовать центры захвата в фосфорах, добавочное поглощение которых не может быть измерено.

\section{§ 3. Некоторые применения метода термического обесцвечивания для исследования центров захвата}

Связь между данными добавочного поглощения и термического высвечивания. Для исследования центров захвата в кристаллах обычно при-

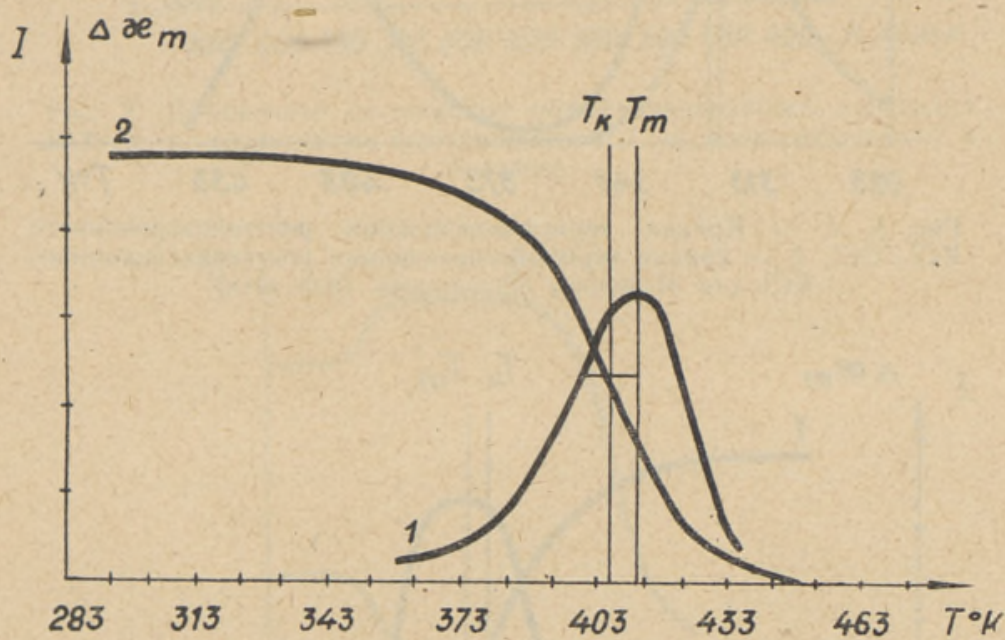

Рнс. 4. 1 - Кривая термовысвечивания $\mathrm{KCl} \cdot \mathrm{TlCl}$ (предварительно прогрет до $\left.380^{\circ} \mathrm{K}\right), 2$ - кривая термообесцвечнвания рентгенизированного $\mathrm{KCl}$ для $F$-полосы поглощения (555 ммк).

меняют метод добавочного поглощения и метод термического высвечивания. Оба метода дают важные данные о центрах захвата. Однако интерпретация полученных результатов обычно затруднена тем, что за исключением нескольких случаев до сих пор не найдены достаточно удобные методы установления взаимосвязи между отдельными полосами добавочного поглощения и определенными пиками термовысвечивания. Такая связь может быть установлена путем облучения возбужденного фосфора светом, соответствующим определенной полосе добавочного поглощения. При этом по селективному ослаблению одного из пиков термовысвечивания может быть найдена связь данного пика и данной полосы добавочного поглощения $\left({ }^{7}\right)$.

Еще более наглядно такая связь может быть установлена методом термического обесцвечивания. На рис. 4 приведена кривая термовысвечивания фосфора $\mathrm{KCl} \cdot \mathrm{TlCl}$ (после предварительного прогрева до $380^{\circ} \mathrm{K}$ ) и кривая термообесцвечивания рентгенизированного кристалла $\mathrm{KCl}$, полученная для $F$-полосы поглощения $(555$ ммк). Из рис. 4 следует, что область резкого спада $\Delta \varkappa_{m}{ }^{F}(T)$ хорошо совпадает с областью пика термо- 
высвечивания. Это позволяет сделать вывод, что пик термовысвечивания при $410^{\circ} \mathrm{K}$ соответствует $F$-центрам захвата. Аналогично рис. 5 , на котором приведены кривые $I(T)$ для $\mathrm{KCl} \cdot \mathrm{TlCl}$ и $\Delta \varkappa_{m}(T)$ для $M$-полосы поглощения в $\mathrm{KCl}$, свидетельствует, что пик термовысвечивания при $325^{\circ} \mathrm{K}$ соответствует $M$-центрам захвата.

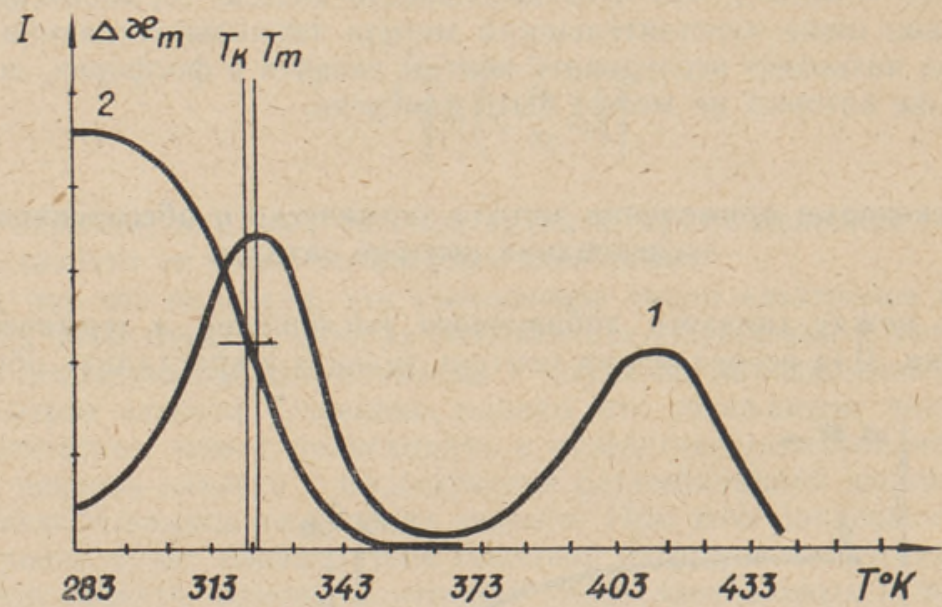

Рис. 5. 1 - Кривая термовысвечивания рентгенизированного $\mathrm{KCl} \cdot \mathrm{TlCl}, 2$ - кривая термообесцвечивания рентгенизированного $\mathrm{KCl}$ для $M$-полосы поглощения ( 825 ммк).

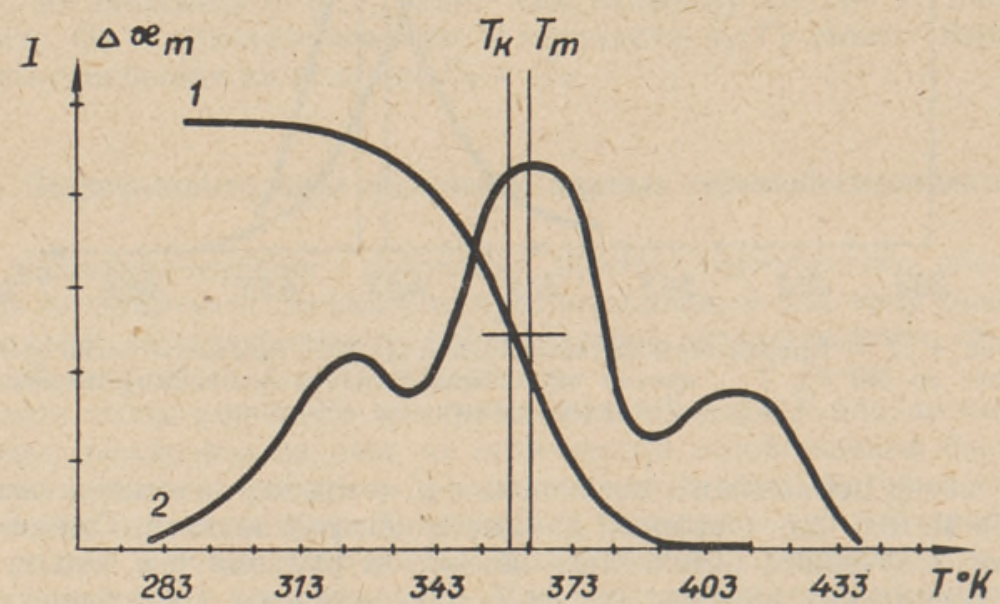

Рнс. 6. $1-$ Кривая термообесцвечивания $\mathrm{KCl} \cdot \mathrm{CaCl}_{2}$ для $Z_{1}$-полосы поглощения (580 ммк), 2 - кривая термовысвечивания рентгенизнроваңного $\mathrm{KCl} \cdot \mathrm{CaCl}_{2} \cdot \mathrm{TlCl}$.

При исследовании примесных центров захвата в $\mathrm{KCl} \cdot \mathrm{CaCl}_{2}$ оказалось, что для полосы добавочного поглощения при 580 ммк, которую Зейтц $\left(^{8}\right)$ приписывает захвату электронов ионами $\mathrm{Ca}^{+}$, кривая термообесцвечивания имеет резкий спад с $T_{k}=360^{\circ} \mathrm{K}$ (рис. 6). Это позволило высказать гипотезу, что в этой области температур в фосфоре $\mathrm{KCl} \cdot \mathrm{CaCl}_{2}$. - $\mathrm{TlCl}$ должен существовать пик термовысвечивания, обусловленный освобождением электронов с ионов $\mathrm{Ca}^{++}$. Эксперимент подтвердил эту гипотезу. В кристалле $\mathrm{KCl} \cdot \mathrm{CaCl}_{2} \cdot \mathrm{TlCl}$ действительно был обнаружен пик термовысвечивания с $T_{m}=365^{\circ} \mathrm{K}$ (рис. 6). 
Итак, метод термообесцвечивания позволяет установить наглядную связь между данными добавочного поглощения и данными термовысвечивания.

Исследование характера кинетики послесвечения. Одной из основных характеристик, определяющих характер кинетики послесвечения в фосфорах, является соотношение между вероятностью рекомбинации $A_{p}$ и вероятностью повторных захватов $A_{3}$. В настоящее время по вопросу о вели-

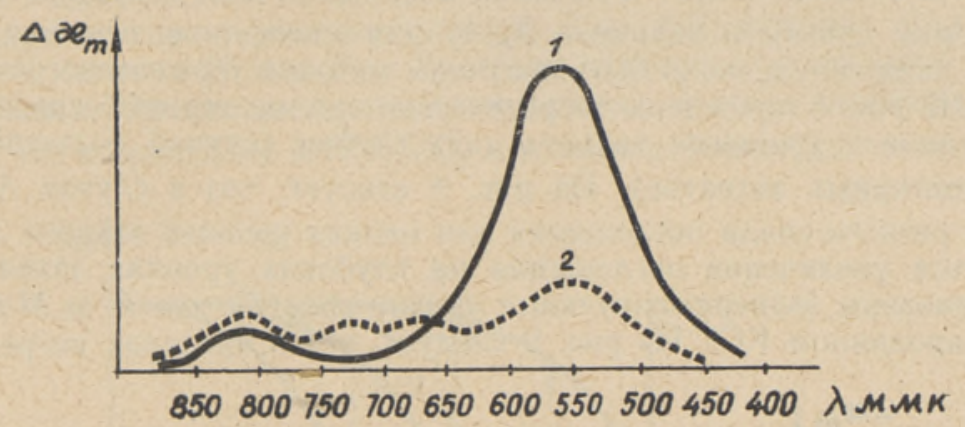

Рис. 7. Добавочное поглощение рентгенизированного кристалла $\mathrm{KCl}: 1$ - сразу после рентгенизацин, 2 - после облучения в $F$-полосе.

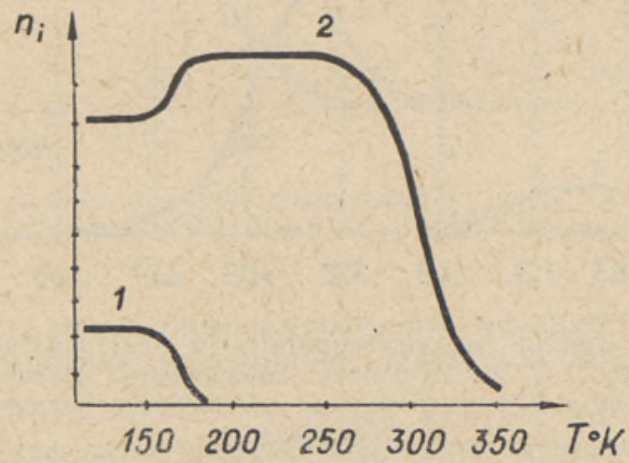

Рис. 8. Теоретические кривые термообесцвечивания для фосфора с уровнями захвата двух сортов для случая большой вероятности повторных захватов $\left(p_{0}=10^{10} \mathrm{ceK}^{-1}\right.$, $\beta_{0}=0,15$ град/сек): 1 - для мелких уровней $E_{T}=0,4$ эв, 2 - для глубоких уровней $E_{T}=0,6$ эв.

чине $A_{p} / A_{3}$ существуют противоречивые точки зрения. Согласно одной из них $A_{3} \gg A_{p}$. Вторая точка зрения (ее придерживается большинство исследователей) предполагает выполнение обратного неравенства $A_{3} \ll A_{p}$. K сожалению, дискуссия о велличине $A_{p} / A_{5}$ очень часто ведется на основании разных умозаключений, а не на основе прямых экспериментальных оценок. Между тем, такие оценки вполне возможны.

Если измерять спектр добавочного поглощения кристалла до ионизации части центров захвата и после оптического или термического высвечивания, то, сравнивая уменьшение одной из полос добавочного поглошения в результате высвечивания в этой полосе $\left(\Delta \varkappa_{1}\right)$ и увеличение дру- 
гих полос поглощения из-за повторных захватов освобождающихся при этом электронов $\left(\Delta \varkappa_{2}\right)$, можно оценить относительную вероятность повторных захватов:

$$
\frac{A_{p}}{A_{3}}=\frac{\Delta x_{1}}{\Delta x_{2}}-1
$$

В случае оптического высвечивания $F$-центров в рентгенизированном кристалле $\mathrm{KCl}$, как следует из рис. $7, \Delta \varkappa_{1} / \Delta \varkappa_{2} \cong 10$, т. е. $A_{p} \gg A_{3}$.

Некоторые данные о величине $A_{p} / A_{3}$ для электронов, освобождаемых тепловым движением, могут быть получены методом термического обесцвечивания. На рис. 8 приведены теоретические кривые термообесцвечивания для кристалла с уровнями захвата двух глубин (случай большой вероятности повторных захватов). Из рис. 8 следует, что в случае $A_{3} \gg A_{p}$ в области резкого спада поглощения для мелких уровней захвата должно наблюдаться увеличение поглощения на глубоких уровнях захвата. На рис. 9 приведены экспериментальные кривые $\Delta \varkappa_{m i}(T)$. для $F$ - и $M$-полос в рентгенизированном $\mathrm{KCl}$. Из рис. 9 следует, что в пределах погрешности

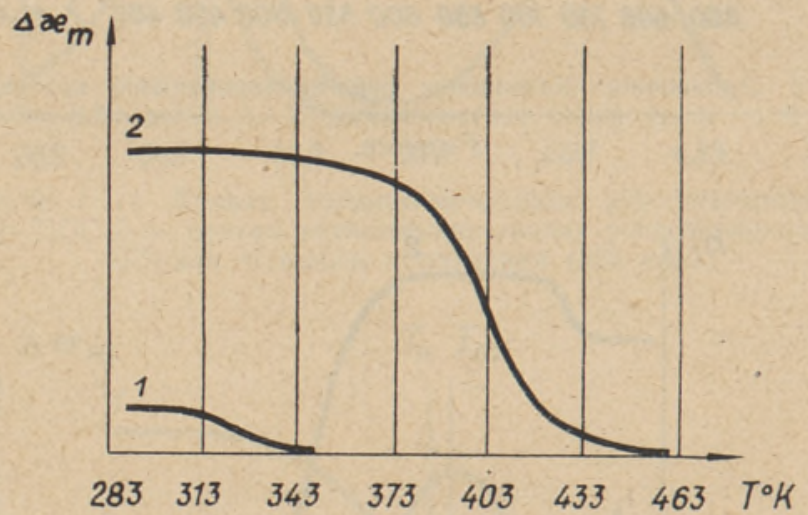

Рис. 9. Кривые термообесцвечивания рентгенизированного $\mathrm{KCl}$ : 1 -в $M$-полосе добавочного поглощения (825 ммк), 2 - в $F$-полосе добавочного поглощения (555 ммк).

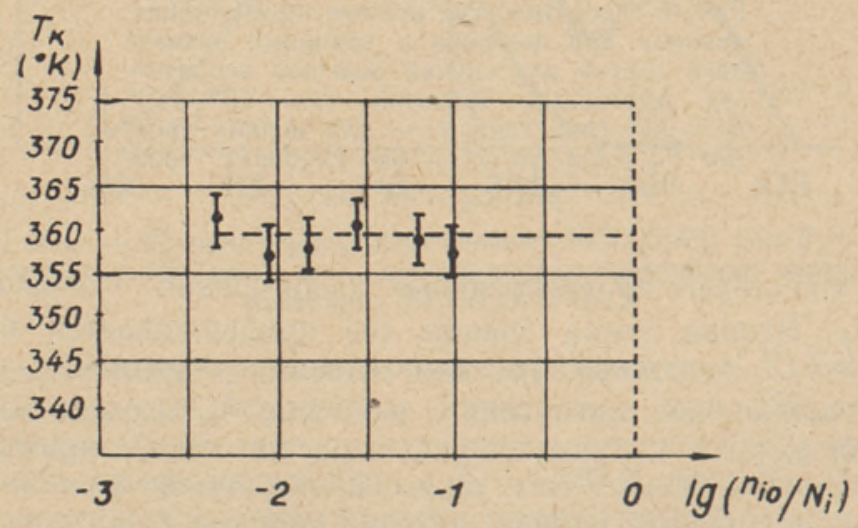

Рис. 10. Зависимость $T_{k}$ кривых термообесцвечивания для $Z_{1}$-полосы поглощения $\left(580\right.$ ммк) в $\mathrm{KCl} \cdot \mathrm{CaCl}_{2}$ от числа запасенных электронов. 
измерений увеличения поглощения на $F$-центрах в области термического разрушения $M$-центров не происходит, что свидетельствует о выполнении для тепловых электронов неравенства $A_{p} \gg A_{3}$.

В $\S 1$ было показано, что по зависимости $T_{k}$ от $n_{0}$ можно оценить величину $\sigma_{3} / \sigma_{p}$. На рис. 10 приведены полученные экспериментально величины $T_{k}$ для кристалла $\mathrm{KCl} \cdot \mathrm{CaCl}_{2}$ (полоса 580 ммк) для различного числа запасенных электронов. В наших опытах $n_{0}$ изменялось почти в 25 раз. Максимальное $n_{0}$ не превышало 0,1 от числа ионов $\mathrm{Ca}^{++}$, служащих в данном случае центрами захвата. Как следует из рис. 10, при уменьшении $n_{0}$ почти в 25 раз $T_{k}$ в пределах погрешности измерения остается неизменным. Сравнение полученных данных с рис. 3 позволяет сделать вывод, что в кристалле $\mathrm{KCl} \cdot \mathrm{CaCl}_{2}$ в условиях наших опытов $\sigma_{3} / \sigma_{p}<0,01$.

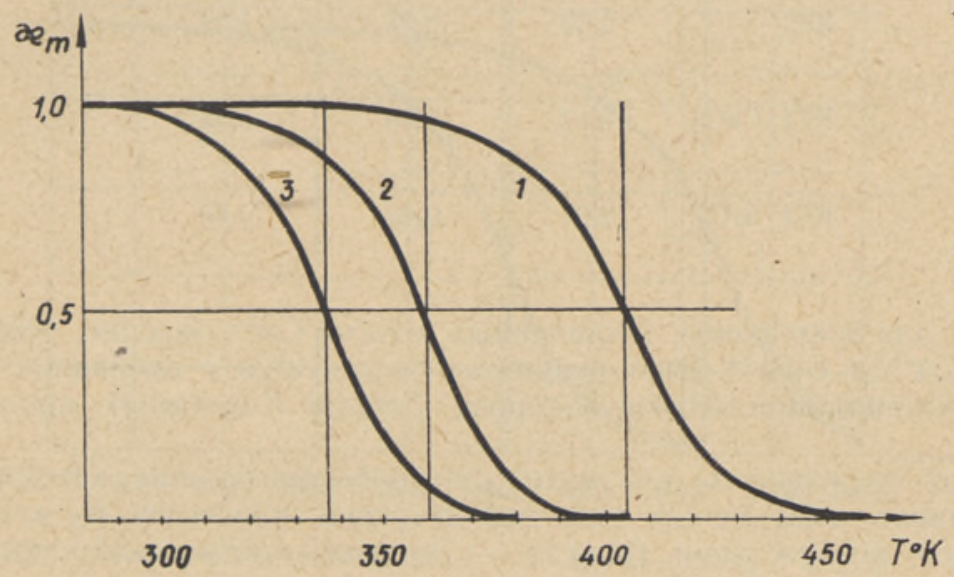

Рис. 11. Кривые термообесцвечивания: $1-$ для $F$-полосы в $\mathrm{KCl}$, 2 - для $Z_{1}$-полосы в $\mathrm{KCl} \cdot \mathrm{CaCl}_{2}, 3$ - для $Z_{1}$-полосы в $\mathrm{KCl} \cdot \mathrm{SrCl}_{2}$.

Таким образом, метод термического обесцвечивания позволяет исследо„вать характер кинетики электронных процессов в кристаллах. Для щелочно-галоидных кристаллов этот метод приводит к выводу, что $A_{3} \ll A_{p} *$.

Определение тепловых энергий ионизации центров захвата. По данным термического обесцвечивания могут быть определены тепловые энергии ионизации центров захвата. Для вычисления $E_{T}$ могут быть использованы соотношения (11) и (15). Еще более удобны для расчетов следующие соотношения, которые могут быть легко получены из (11) и (15) и условня $n_{i k}=0,5 \cdot n_{i 0}$ :

$$
\begin{aligned}
& \text { для } A_{3} \gg A_{p} \quad E_{T}=\frac{2 k T_{k}^{2}}{\delta_{k}}, \\
& \text { для } A_{3} \ll A_{p} \quad E_{T}=\frac{k T_{k}^{2}}{\delta_{k}} .
\end{aligned}
$$

На рис. 11 приведены кривые термообесцвечивания для $F$-центров в $\mathrm{KCl}$ и $\mathrm{Z}_{1}$-полос поглощения в $\mathrm{KCl} \cdot \mathrm{CaCl}_{2}$ и $\mathrm{KCl} \cdot \mathrm{SrCl}_{2}$. По получен-

* Отметим, что $A_{3} \ll A_{p}$ даже при отсутствии полного заполнения уровней захвата электронами. Вероятно, малая величина $A_{3}$ обусловлена тем, что электроны захватываются блнжайшими к центрам свечения центрами захвата. 
ным данным были рассчитаны тепловые энергии ионизации соответствующих центров захвата. Для расчетов использовались соотношения (15) и (19). В обоих случаях, как это следует из таблицы 1 , получены близкие результаты. $E_{T}$, определенные по $\Delta \varkappa_{m}(T)$ для $F$-центров, хорошо согласуются с данными Смакулы, полученными при исследовании электропроводности аддитивно окрашенных кристаллов $\mathrm{KCl}\left({ }^{9}\right)$, и данными, полученными нами методом термического высвечивания $\left({ }^{10}\right)$.

Таблица 1

\begin{tabular}{l|c|c|c}
\hline & $\begin{array}{c}E_{2} \\
\text { эв }\end{array}$ & $\begin{array}{c}E_{T} \\
\text { По (15) } \\
\text { эв }\end{array}$ & $\begin{array}{c}E_{T} \\
\text { по }(19) \\
\text { эв }\end{array}$ \\
\hline $\mathrm{KCl}$ & 2,22 & 0,97 & 1,02 \\
\hline $\mathrm{KCl} \cdot \mathrm{CaCl}_{2}$ & 2,12 & 0,85 & 0,86 \\
\hline $\mathrm{KCl} \cdot \mathrm{SrCl}_{2}$ & 2,08 & 0,80 & 0,80 \\
\hline
\end{tabular}

$E_{T} \mathrm{Ca}^{++}$для электронов, захваченных ионами $\mathrm{Ca}^{++}$, меньше, чем $E_{T} \mathrm{Sr}^{++}$; $E_{T}{ }^{\mathrm{F}}>E_{T} \mathrm{Ca}^{++}>E_{T} \mathrm{Sr}^{++}$. Әтот результат согласуется с величиной ионизационных потенциалов атомов водорода (аналог $F$-центров), ионов $\mathrm{Ca}^{+}$и $\mathrm{Sr}^{+}$.

Следует подчеркнуть, что метод термообесцвечивания автоматически устанавливает связь между тепловой энергией ионизации $E_{T}$ и энергией оптического возбуждения (иногда - энергией оптической ионизации) $E_{\lambda}$ центров захвата. Величины $E$, для исследованных случаев приведены в табл. 1. Сравнение $E_{T}$ и $E_{\lambda}$ показывает, что $E_{\lambda} \geqslant 2 E_{T}$. Причины такого резкого различия тепловых и оптических энергий ионизации обсуждены в другой статье автора $\left({ }^{10}\right)$.

Обнаружение активаторных центров захвата в области температурного тушения. В последние годы широкое распространение получила гипотеза о том, что электронные центры захвата в фосфорах могут создаваться ионами активирующей примеси (11).

В щелочно-галоидных кристаллах «активаторные» центры захвата были обнаружены методом добавочного поглощения около 20 лет тому назад $\left({ }^{12}\right)$. Нами было показано $\left({ }^{7}\right)$, что на кривой термовысвечивания $\mathrm{KCl} \cdot \mathrm{TlCl}$ имеется пик, отсутствующий в $\mathrm{KCl} \cdot \mathrm{AgCl}$ и $\mathrm{KCl} \cdot \mathrm{CuCl}$. При этом мы полагали, что этот пик термовысвечивания соответствует захвату электронов ионами $\mathrm{Tl}^{+}$. Так как атомы $\mathrm{Ag}^{0}$ и $\mathrm{Cu}^{0}$ имеют еще более высокие потенциалы ионизации, чем атомы $\mathrm{Tl}^{0}$, то можно было надеяться обнаружить в $\mathrm{KCl} \cdot \mathrm{CuCl}$ и $\mathrm{KCl} \cdot \mathrm{AgCl}$ «медные» и «серебряные» пики термовысвечивания. Опыт, однако, дал отрицательный результат. Была высказана гипотеза, что центры захвата, обусловленные присутствием ионов $\mathrm{Ag}^{+}$и $\mathrm{Cu}^{+}$, не могут быть обнаружены методом термовысвечивания из-за наличия сильного температурного тушения. Данные термообесцвечивания подтвердили это предположение. Для «серебряной» полосы добавочного поглощения в $\mathrm{KCl} \cdot \mathrm{AgCl}$ (340 ммк) $T_{k}$ кривой термообесцвечивания значительно больше, чем $T_{k}$ для $F$-полосы (рис. 12$)$. Между тем, даже в области $F$-пика термовысвечивания уже имеется сильное температурное тушение. Итак, действительно, тушение не позволяет обнаружить методом термовысвечивания глубокие уровни захвата. 
Метод термообесцвечивания обладает более широкими пределами применимости и позволяет определить $E_{T}$ этих уровней. Как и следовало ожидать, из эксперимента следует, что $E_{T} \mathrm{Ag}^{+}>E_{T} \mathrm{Tl}^{+}$.

Приведенные выше данные, несмотря на их иллюстративный характер, служат, по нашему мнению, свидетельством в пользу плодотворности применения метода термического обе̨сцвечивания для исследования центров захвата в кристаллах. Применение этого метода в комбинации с методом термовысвечивания и методом добавочного поглощения поможет расширить наши знания о центрах захвата в кристаллических телах.

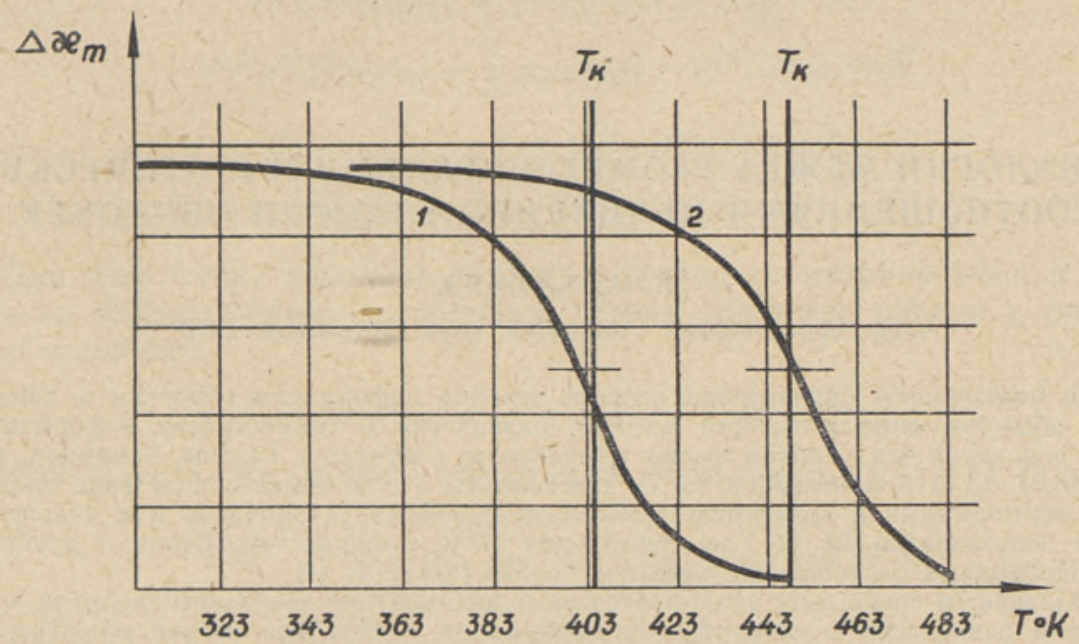

Рис. 12. Кривые термообесцвечивания: $t$ - для $F$-полосы в $\mathrm{KCl}, 2$ - для «серебряной» полосы (340 ммк) в $\mathrm{KCl} \cdot \mathrm{AgCl}$.

Автор приносит глубокую благодарность действительному члену АН ЭССР Ф. Д. Клементу за обсуждение полученных результатов, а также Н. Е. Лущик и Э. Г. Вильпер за помощь в работе.

Ннститут физики и астрономии

Академии наук Эстонской ССР

\section{JИТЕРАТУРА}

1. R. B u b e, Phys. Rev., 83, 393, 1951.

2. G. Garlick, F. Gibson, Proc. Roy. Soc., 188, 485, 1947.

3. Н. П. К ал аб у хо в, Изв. АН СССР, Серия физич. 16, 130, 1952.

4. D. Dutton, R. M a urer, Phys. Rev., 90, 126, 1953.

5. С. И. Пека р, Исследования по электронной теории кристаллов, ГИТТЛ, 1951.

6. Ф. Д. Кле м ен т, Изв. АН СССР, Серия физич., 9, 411, 1945.

7. Ч. Б. Л у щ и к, Изв. АН СССР, Серия физич., $18,687,1954$.

8. F. S e it z, Phys. Rev., 83, 134, 1951.

9. A. S m a c u la, Gött. Nachr., 1, 55, 1934.

10. Ч. Б. Л у щ и к, ДАН СССР, 101, 833, 1955.

11. В. Л. Л е в и и н, Изв. АН СССР, Серия физич., 9, 355, 1945.

12. А. С. Т о п о ре ц. ДАН СССР, 4, 25, 1935. 